\title{
UTILIZAÇÃO DO VÉRTICE DE MENOR ENCARGO PARA OTIMIZAÇÃO DE ROTA A SER PERCORRIDA
}

\author{
Lohane Viterbo de Carvalho \\ Centro Universitário Augusto Motta \\ Av. Cesário de Melo, 2541 - Campo Grande, Rio de Janeiro - RJ, 23052-102 \\ lohaneviterbo@yahoo.com.br
}

\section{RESUMO}

Esse trabalho tem como objetivo principal analisar as rotas a serem percorridas durante o transporte dos colaboradores de uma indústria para atendimento de uma parada programada para a manutenção de um carregador de navio, fazendo com que essa logística funcione de forma otimizada durante as viagens, tendo a pesquisa operacional como aliada durante esse estudo, utilizando o método do caixeiro viajante (menor encargo) em um problema de transporte e de rede de distribuição, a fim de encontrar o menor caminho para a redução dos custos de transporte e melhoria da eficiência do serviço.

Palavra-chave: Otimização; Pesquisa Operacional; rotas.

\begin{abstract}
This work has as main objective to analyze the routes to be traveled during the transport of the employees of an industry to attend a scheduled stop for the maintenance of a ship loader, making that logistics work optimally during the trips, having the operational research as an ally during this study, using the traveling salesman's method (lower burden) on a transportation and distribution network problem, in order to find the smallest path to reduce transport costs and improve service efficiency.
\end{abstract}

Keywords: Optimization; Operational Research; routes.

\section{Como Citar:}

VITERBO, Lohane. Utilização do vértice de menor encargo para otimização de rota. In: SIMPÓSIO DE PESQUISA OPERACIONAL E LOGÍSTICA DA MARINHA, 19., 2019, Rio de Janeiro, RJ. Anais [...]. Rio de Janeiro: Centro de Análises de Sistemas Navais, 2019.

\section{INTRODUÇÃO}

Pode-se observar a crescente busca das grandes, médias e pequenas empresas pelo seu referencial no mercado de trabalho, isso se dá pela grande competitividade existente no cenário atual.

Dessa forma, a pesquisa operacional chegou para facilitar a vida desses empresários através de técnicas e métodos matemáticos voltados para otimização dos recursos existentes dentro dessas organizações auxiliando em problemas de diferentes seguimentos. 
Para Moreira (2010, p.3) “A pesquisa operacional lida com problemas de como conduzir e coordenar certas operações em uma organização, e tem sido aplicada a diversas áreas, tais como indústrias, transportes, telecomunicações, finanças, saúde, serviços públicos, operações militares etc."

A utilização da PO ainda é muito criticada, Para Arnales (2015),

\begin{abstract}
A denominação Pesquisa Operacional é comumente motivo de críticas e reflexões, pois não revela a abrangência da área e pode dar falsa impressão de estar limitada à análise de operações. Alguns autores sugerem outras denominações preferíveis, por exemplo, a análise de decisões, mas o termo pesquisa operacional é bastante difundido no âmbito das engenharias (em particular, da engenharia de produção), ciências de computação, matemática, entre outros (ARNALES, 2015, p. 3).
\end{abstract}

A Pesquisa Operacional é um método que visa e conseguir resultados eficazes e confiáveis nas atividades de uma organização. É um eficiente modo de apresentar uma melhoria no processo. Dentro dela, pode-se encontrar várias ferramentas de aplicação para um melhor resultado de forma simples e eficaz.

\title{
2. METODOLOGIA
}

A estratégia metodológica que orienta este estudo tem o objetivo de otimizar as rotas a serem percorridas durante o transporte dos colaboradores de uma determinada indústria através da técnica conhecida da Pesquisa Operacional chamada de método de inserção do vértice de menos encargo, que identifica uma "rota ótima" a se percorrer otimizando o tempo.

Referente aos conceitos e terminologias utilizou-se pesquisa bibliográfica e pesquisa descritiva, sendo a pesquisa bibliográfica a partir de livros, artigos científicos, artigos online e demais fonte de consulta, além disto, será apresentado um estudo de caso de uma indústria fictícia, servindo de fonte de dados para a elaboração deste trabalho.

\section{PESQUISA OPERACIONAL}

Desde o início da Revolução Industrial pode-se observar o crescimento exponencial das organizações com o passar dos anos. Dessa forma as pequenas empresas evoluíram para as grandes corporações de renome atualmente. Um dos fatores responsáveis por essa evolução foi a divisão do trabalho e das responsabilidades gerenciais nesses estabelecimentos. $\mathrm{O}$ resultado disso é o que podemos ver atualmente com empresas alcançando resultados incríveis.

Por outro lado, como tudo possui o seu ponto negativo e positivo, esse grande desenvolvimento acabou resultando em novos problemas a serem resolvidos e que até hoje é motivo de dor de cabeça para muitos empresários. Um dos problemas decorrentes disso é que à medida que um processo fica mais complexo, mais torna-se complicado a alocação dos recursos disponíveis para as atividades de maneira mais ótima e eficiente para toda a organização. Esses tipos de problemas e a necessidade de encontrar o melhor caminho para solucioná-los criaram condições necessárias para o surgimento da Pesquisa Operacional (comumente referida como PO).

A técnica de pesquisa operacional se desenvolveu na Inglaterra, com Blackett dirigindo um grupo de especialistas dedicados à análise de operações militares. As análises procuravam maximizar os escassos recursos disponíveis para as operações militares. Ao final da guerra, as técnicas passaram a serem aplicadas nos meios empresariais da Inglaterra e dos Estados Unidos. (PEINADO, 2007). Dessa forma, iniciou o conceito de programação linear. 
Um problema de programação linear (PL) consiste na maximização ou minimização de uma função linear de várias variáveis, designada por uma função objetivo, em que as variáveis estão sujeitas a um conjunto de restrições também lineares. As variáveis de decisão representam, em geral, níveis de atividades, as restrições podem resultar de limitações na disponibilidade de recursos ou de requerimentos mínimos que devem ser atingidos e a função objetivo representa uma medida do desempenho do sistema. (CLÍMACO, 2003.)

Quando se procura refletir sobre uma situação real, na tentativa de explicar, de entender ou de agir sobre ela, um modelo matemático pode surgir. Este modelo em geral traz simplificações que nem sempre condizem com a realidade. Assim, de modo geral um modelo matemático retrata uma situação idealizada da realidade, simplificada o bastante para permitir cálculos matemáticos, e tem como objetivo o entendimento do fenômeno e possíveis previsões de seu comportamento futuro (PASSOS, 2009).

Apesar de ser um método que oferece facilidade ao encontrar soluções ótimas para problemas complexos, deve-se atentar sempre para o primeiro passo a ser tomado durante o reconhecimento do problema que consiste em determinar as variáveis de decisão, pois é só a partir delas que as inequações/equações podem ser determinadas.

\subsection{PROBLEMA DO CAIXEIRO VIAJANTE (PCV)}

Em tempos passados, quando não havia recursos suficientes de transporte, os caixeiros viajantes realizavam o papel de transportar produtos para diferentes cidades a fim de comercializa-los em locais onde não se havia disponibilidade.

Devido a grande dificuldade existente para a execução desse trabalho, os caixeiros viajantes utilizavam do conhecimento empírico para as tomadas de decisões que tinham que lidar ao longo de cada jornada. Dessa forma, sem nenhum método que o auxiliasse, o caixeiro viajante sabendo todos os locais em que deveria visitar, deveria fazer uma estimativa da distância a ser percorrida em cada local a fim de tentar otimizar sua viagem.

Com o passar do tempo, tipos de problemas como esse foram estudados, e com o avanço do método científico, foram desenvolvidas inúmeras ferramentas a fim de auxiliar nas decisões a serem tomadas.

O problema do caixeiro viajante pode ser definido como um método capaz de otimizar uma rota quando se possui muitos locais a serem visitados somente uma vez, retornando novamente ao seu ponto inicial.

\subsubsection{Método do vértice de menor encargo}

Trata-se de um algoritmo utilizado na inserção de vértices a cada iteração, ou seja, escolhe-se um par de vértices que formam o subciclo de menor distância total. Dessa forma, insere-se o vértice escolhido de modo a minimizar o aumento da distância total.

Esse procedimento é repetido no subciclo corrente até completar o ciclo.

\section{DESCRIÇÃO DO PROBLEMA}

A equipe do PCM de uma indústria negociou com a equipe de PCP a parada programada para troca do motor de acionamento da lança de um dos carregadores de navio para o final de semana. Por esse motivo, foi necessário escalar alguns colaboradores da equipe de manutenção preventiva para essa data.

Essa empresa deseja que a logística funcione de forma otimizada, durante o transporte desses colaboradores. O propósito para a otimização seria a obtenção de um caminho "quase ótimo" a percorrer durante o percurso, utilizando o método de inserção do vértice de menor encargo. 
Esse método funciona como uma ferramenta que é capaz de definir o melhor caminho a se percorrer considerando uma listagem grande de destinos a se chegar.

\subsection{APLICAÇÃo do MÉTOdO DE INSERÇão dO VÉRTICE DE MENOR ENCARGO PARA O ESTUDO DO CASO}

Uma vez apresentado o problema, fica fácil entender que estamos procurando o caminho "quase ótimo" que satisfaçam a otimização durante o percurso sendo da saída ao retorno até a indústria.

\begin{tabular}{|l|c|}
\hline \multicolumn{2}{|c|}{ Localização } \\
\hline A) & Colaborador 1 - Campo Grande \\
B) & Indústria "X" - Ilha da Madeira \\
C) & Colaborador 2 - Itaguaí \\
D) & Colaborador 3 - Santa Cruz \\
E) & Colaborador 4 - Paciência \\
\hline
\end{tabular}

A partir de então, chegamos a formação da matriz booleana com as respectivas distâncias de um local para o outro.

\begin{tabular}{|c|c|c|c|c|c|}
\cline { 2 - 6 } \multicolumn{1}{c|}{} & A & B & C & D & E \\
\hline A & & 37 & 11 & 23 & 33 \\
\hline B & 38 & & 28 & 19 & 8 \\
\hline C & 12 & 29 & & 15 & 25 \\
\hline D & 26 & 19 & 11 & & 9 \\
\hline E & 36 & 8 & 26 & 10 & \\
\hline
\end{tabular}

Aplica-se então o método no grafo orientado com a matriz de distâncias (KM) conforme visto anteriormente, é feito a escolha do circuito inicial.

\begin{tabular}{|c|c|c|c|c|}
\hline A & $\mathrm{ABA}=75$ & $\mathrm{ACA}=23$ & $A D A=49$ & $A E A==69$ \\
\hline B & & $\mathrm{BCB}=57$ & $\mathrm{BDB}=38$ & $\mathrm{BEB}=16^{*}$ \\
\hline C & & & $C D C=26$ & $C E C=51$ \\
\hline D & & & & $\mathrm{DED}=19$ \\
\hline
\end{tabular}


- $\quad \mathrm{BEB}=16 \mathrm{~km}-$ possíveis inserções no par B,E ou no par E,B.

\begin{tabular}{|c|c|c|}
\hline Vértice inserido & $\begin{array}{c}\text { Aumento de distância } \\
\text { de B para E }\end{array}$ & $\begin{array}{c}\text { Aumento de distância } \\
\text { de E para B }\end{array}$ \\
\hline A & $38+33-8=63$ & $36+37-8=65$ \\
\hline C & $28+25-8=45$ & $26+29-8=47$ \\
\hline D & $19+9-8=20$ & $10+19-8=21$ \\
\hline
\end{tabular}

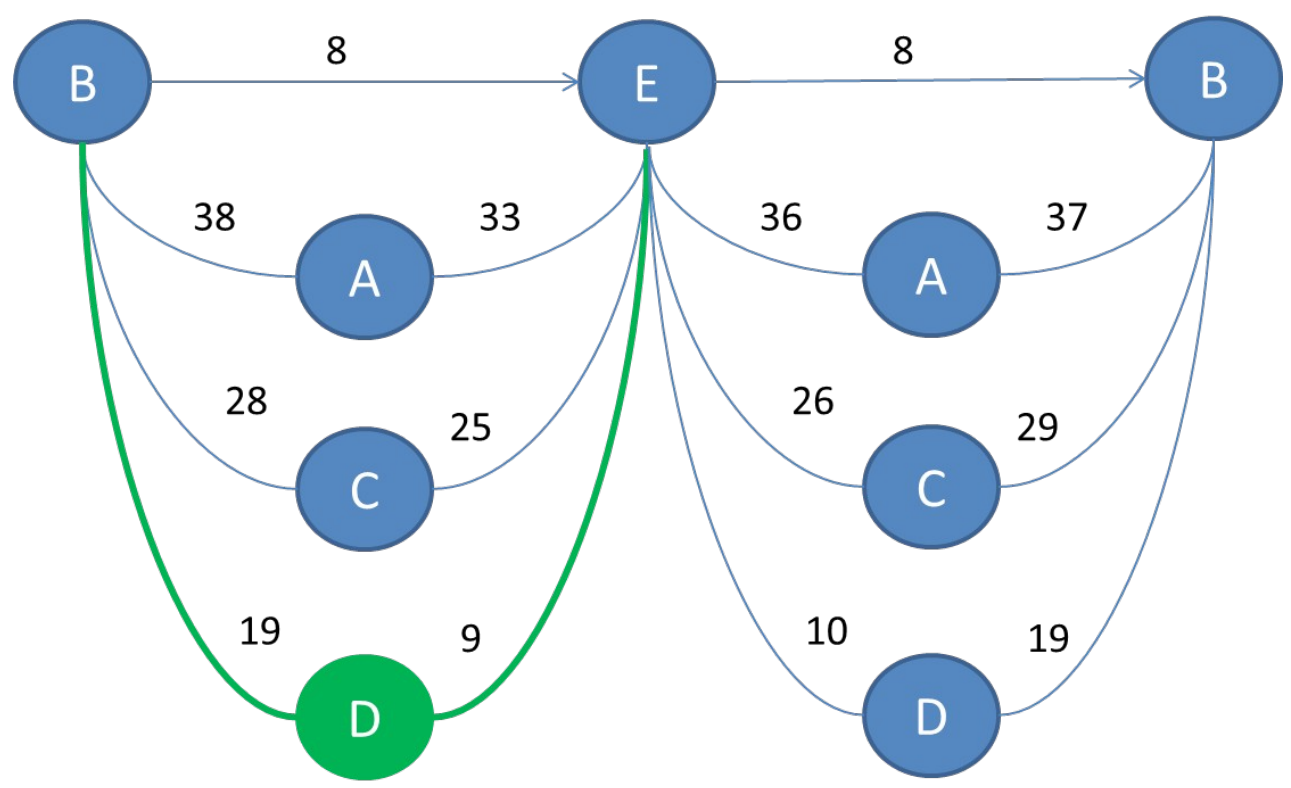

Então, o subcircuito BDEB com distância total de $36 \mathrm{~km}$ (subcircuito anterior de 16 $\mathrm{km}$, aumentando com $20 \mathrm{~km}$ ). Repete-se então o mesmo processo com o novo subcircuito formado.

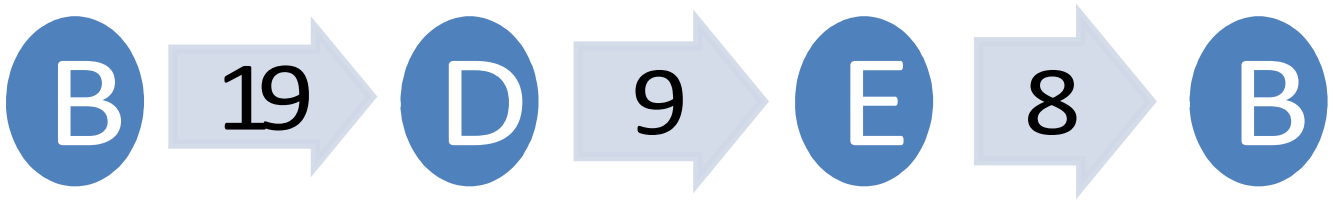

- $\quad$ BDEB = $36 \mathrm{~km}-$ possíveis inserções no par B,D, no par D,E ou no par E,B.

\begin{tabular}{|c|c|c|c|}
\hline Vértice inserido & $\begin{array}{c}\text { Aumento de distância } \\
\text { de B para D }\end{array}$ & $\begin{array}{c}\text { Aumento de distância } \\
\text { de D para E }\end{array}$ & $\begin{array}{c}\text { Aumento de } \\
\text { distância de E para B }\end{array}$ \\
\hline A & $38+23-19=42$ & $26+33-9=50$ & $36+37-8=65$ \\
\hline C & $28+15-19=24$ & $11+25-9=27$ & $26+29-8=47$ \\
\hline
\end{tabular}




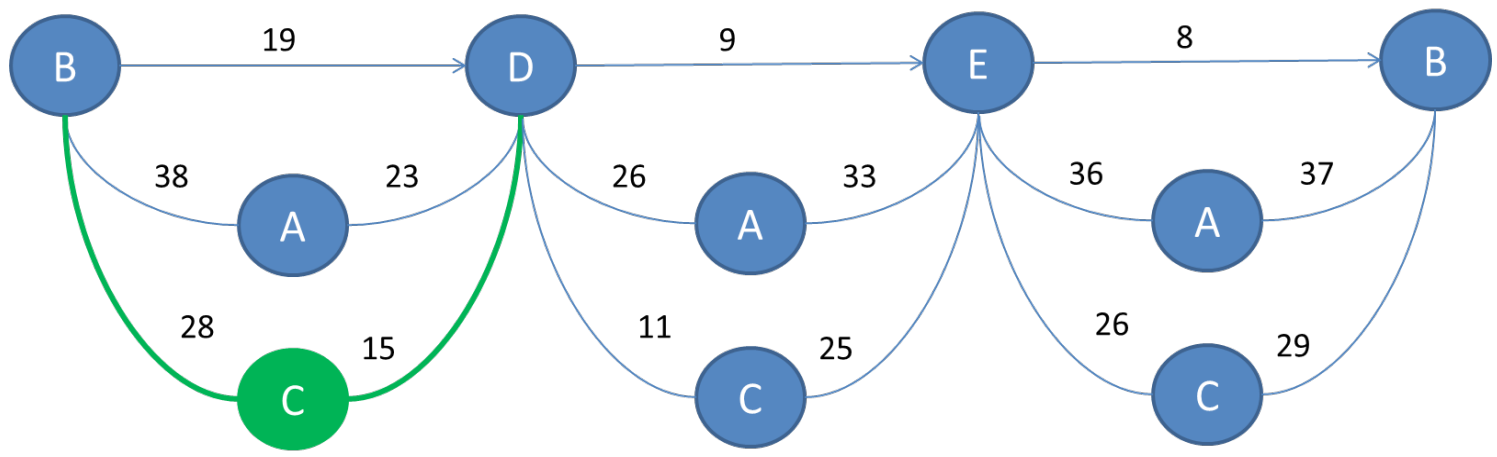

Então, o subcircuito BCDEB com distância total de $60 \mathrm{~km}$ (subcircuito anterior de 36 $\mathrm{km}$, aumentando com $24 \mathrm{~km}$ ). Repete-se então o mesmo processo com o novo subcircuito formado.

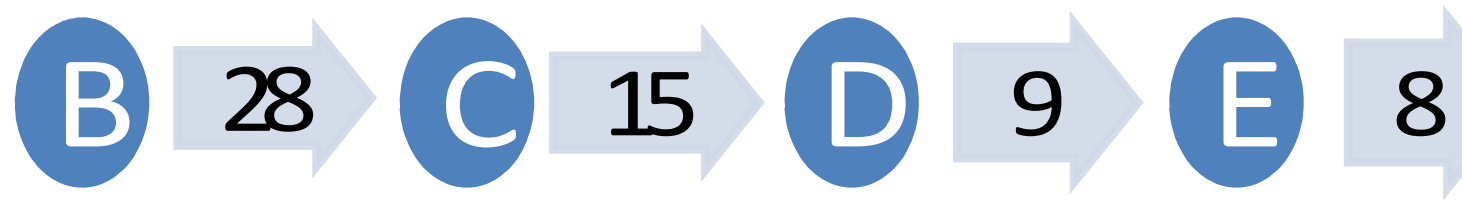

- $\mathrm{BCDEB}=60 \mathrm{~km}$ - possíveis inserções no par B,C, no par C,D, no par D,E, ou no par E,B.

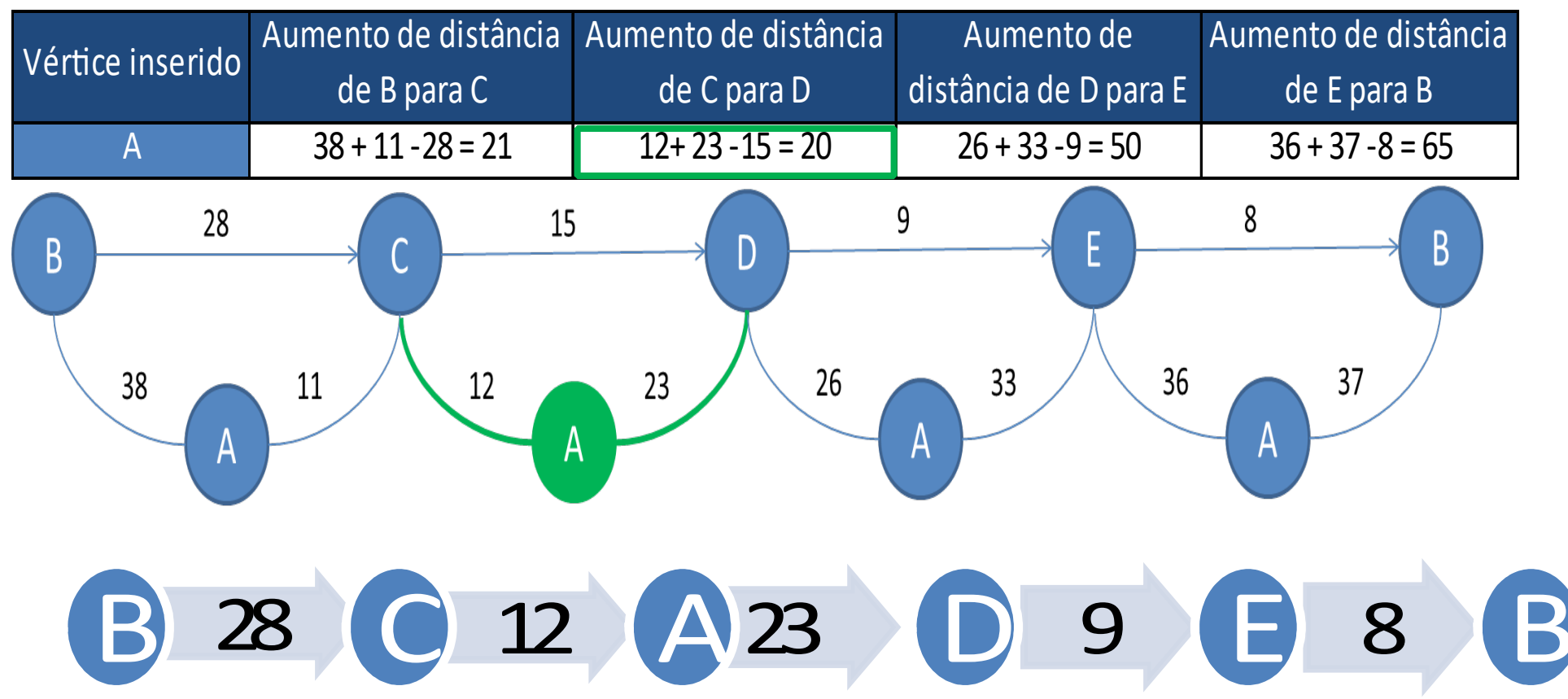

Por fim, obtem-se o subcircuito BCADEB com distância total de $80 \mathrm{~km}$ (subcircuito anterior de $68 \mathrm{~km}$, aumentando com $20 \mathrm{~km}$ ).

\section{CONCLUSÃO}

Conforme pode ser observado no decorrer deste trabalho e no estudo de caso apresentado, o objetivo principal foi atingido, ou seja, através do método utilizado foi possível encontrar o menor percurso (BCADEB) a se seguir para o transporte dos colaboradores escalados para a atividade de manutenção no final de semana com o total de $80 \mathrm{~km}$ a ser percorrido. 
Não obstante a existência de outras ferramentas disponíveis na literatura para a utilização, a ferramenta escolhida e utilizada para a realização desse trabalho foi constatada que seria a mais adequada para expor de forma clara e objetiva o problema exposto.

Dado exposto pode-se concluir a eficácia deste trabalho através dos resultados apresentados, mostrando os benefícios do correto uso desse método do vértice de menor encargo para a otimização de rotas a serem percorridas, minimizando o tempo gasto durante uma viagem. Utilizando um método para a solução de problemas simples e eficiente, foi possível identificar uma rota ótima a ser seguida.

\section{REFERÊNCIAS BIBLIOGRÁFICAS}

[1] ARENALES, Marcos et al. Pesquisa operacional: para cursos de engenharia. Elsevier Brasil, 2015.

[2] CLÍMACO, João Namorado; et al. Programação linear multiobjectivo: do modelo de programação linear clássico à consideração explícita de várias funções objetivo. Imprensa da Universidade de Coimbra/Coimbra University Press, 2003.

[3] MOREIRA, D.A. Pesquisa Operacional: Curso Introdutório. São Paulo: Thomson Learning, 2010.

[4] PASSOS, Adão Nascimento dos et al. Estudos em programação linear. 2009.

[5] PEINADO, Jurandir; et al. Administração da produção. Operações industriais e de serviços. Unicenp, 2007.

As Referências Bibliográficas devem seguir as normas da ABNT/NBR 6023. 